\title{
SIMPLE AND SUBDIRECTLY IRREDUCIBLES BOUNDED DISTRIBUTIVE LATTICES WITH UNARY OPERATORS
}

\author{
SERGIO ARTURO CELANI
}

Received 27 February 2005; Revised 15 July 2006; Accepted 18 July 2006

We characterize the simple and subdirectly irreducible distributive algebras in some varieties of distributive lattices with unary operators, including topological and monadic positive modal algebras. Finally, for some varieties of Heyting algebras with operators we apply these results to determine the simple and subdirectly irreducible algebras.

Copyright (c) 2006 Hindawi Publishing Corporation. All rights reserved.

\section{Introduction}

Distributive lattices with operators (DLO) are a natural generalization of the notion of Boolean algebras with operators. An operator in a bounded distributive lattice $A$ is a function $f: A^{n} \rightarrow A$ which preserves $\wedge$ (or $\vee$ ) in each coordinate.

In the last few years these classes of algebras have been actively investigated since they appear as algebraic counterpart of many logics. Some important contributions in this area have been the papers of Goldblatt [12], Petrovich [16], and Sofronie-Stokkermans [18] which deal with the representation and topological duality for DLO. More recently, in [11] Gehrke et al. have studied conditions for canonicity and an automatic mechanism for the translation of equations that are Sahlqvist. In [17] Sofronie-Stokkermans studies a uniform presentation of representation and decibility results related to a Kripke-style semantics, and the link between algebraic and Kripke-style semantics of several nonclassical logics.

Positive modal logic was introduced by Dunn in [10], and it corresponds to the positive fragment of the local modal consequence relation defined by the class of all Kripke frames. The algebraic semantic of this fragment is the variety of positive modal algebras (or PM-algebras) introduced in [10], and further studied by means of topological methods in [7], and in [6] by methods from abstract algebraic logic. A PM-algebra is a bounded distributive lattice with two unary modal operators $\square$ and $\diamond$ satisfying additional conditions that relate to these operators.

Topological Boolean algebras or closure Boolean algebras were given by McKinsey and Tarski [15] to conduct an algebraic study of topological spaces (see also [4]). In [13],

Hindawi Publishing Corporation

International Journal of Mathematics and Mathematical Sciences

Volume 2006, Article ID 21835, Pages 1-20

DOI 10.1155/IJMMS/2006/21835 
Halmos introduced monadic Boolean algebras for an algebraic study of the one variable fragment of predicate logic. Important classes of bounded distributive lattices with operators that generalize the monadic Boolean algebras are the Q-distributive lattices introduced by Cignoli [9], and the monadic Heyting algebras studied by Bezhanishvili [2].

In research on DLO, Priestley duality is a useful tool. The dual space associated with a DLO is a Priestley space with a $(n+1)$-ary relation for each $n$-ary operator (see $[12,18])$. The dual spaces of PM-algebras can be defined with a unique binary relation or with two binary relations, but strongly related between them (see [7]). In Birchall's Master's thesis [3] there is a duality for perfect distributive lattices with unary operators, and there are some results on subdirect irreducibility using topological duality.

Since any variety of algebras is determinated by the subdirectly irreducible algebras, it is important to have a characterization of them in varieties of (DLO). The main contribution of this paper aims at giving a characterization of simple and subdirectly irreducible of certain varieties of positive modal algebras, and certain varieties of Heyting algebras with unary operators.

The paper is structured as follows: in Section 2 we start by recalling some basic definitions and results on the Priestley duality for distributive lattices with unary operators. This duality has been developed in $[12,18]$ for the general case, in [16] for the case of distributive lattices with an operator of type $\diamond$, and in [7] for positive modal algebras. In Section 3, we will give a topological characterization of congruences and we will determine the simple and subdirectly irreducible algebras for some varieties of distributive lattices with unary operators of type $\square$ and $\diamond$. In Section 4 we will introduce the variety of topological positive modal algebras (TPM-algebras) and the variety of monadic positive modal algebras (MPM-algebras) as a generalization of the closure Boolean algebras and monadic Boolean algebras, respectively. The TPM-algebras and MPM-algebras can be considered as the algebraic semantics of the positive fragment of the local modal consequence relation defined by the class of all Kripke frames $\langle X, R\rangle$, where the binary relation $R$ is reflexive and transitive, and $R$ is an equivalence, respectively. It is interesting to note that the characteristic axiom $\square(a \vee b) \leq \square a \vee \diamond b$, valid in positive modal algebras, is not true in monadic Heyting algebras, thus the $\rightarrow$-free reduct of a monadic Heyting algebra is not an MPM-algebra. In Section 5 we will study the simple and subdirectly irreducible algebras in some varieties of Heyting algebras with modal operators $\square$ and $\diamond(\square \diamond$-Heyting algebras). Some related results on Heyting algebras with operators appear in [14] (see also [19]). We will prove that every $\square$-congruence in $\square \diamond$-Heyting algebras is also a $\diamond$ congruence, but there exists $\diamond$-congruences that are not $\square$-congruences. This shows a certain asymmetry between the modal operators $\square$ and $\diamond$ when considering Heyting algebras. This fact has also been remarked by Bezhanishvili in [2], and by Božić and Došen in $[5]$.

\section{Preliminaries}

Let $\langle X, \leq\rangle$ be a poset. The set of all increasing subsets of $X$ is denoted by $\mathscr{P}_{i}(X)$. It is clear that $\mathscr{P}_{i}(X)$ is a bounded distributive lattice under the operations $\cup$ and $\cap$. A totally order-disconnected topological space is a triple $\langle X, \leq, \tau\rangle$ such that $\langle X, \leq\rangle$ is a poset, $\langle X, \tau\rangle$ is a topological space, and given $x, y \in X$ such that $x \not \leq y$ there is a clopen increasing set 
$U$ such that $x \in U$ and $y \notin U$. A Priestley space is a compact totally order-disconnected topological space. If $X$ is a Priestley space, the set of all clopen increasing sets of $X$ is denoted by $D(X)$. Since $D(X)$ is a ring of sets, then $\langle D(X), \cup, \cap, \varnothing, X\rangle$ is a bounded distributive lattice. It can be proved that $D(X)$ is a subbasis for the topology $\tau$.

If $\mathbf{A}=\langle A, \vee, \wedge, 0,1\rangle$ is a bounded distributive lattice and $X(\mathbf{A})$ is the set of all prime filters of $A$, then $X(\mathbf{A})$ is a Priestley space with inclusion as order and with the topology having as subbasis sets of the form $\sigma_{\mathbf{A}}(a)=\{P \in X(\mathbf{A}): a \in P\}$ and $X(\mathbf{A}) \backslash \sigma_{\mathbf{A}}(a)=\sigma_{\mathbf{A}}(a)^{c}$ for each $a \in A$. It is known that the map $\sigma_{\mathbf{A}}: \mathbf{A} \rightarrow D(X(\mathbf{A}))$ is a lattice isomorphism.

If $\langle X, \leq, \tau\rangle$ is a Priestley space, then the mapping $F_{X}: X \rightarrow X(D(X))$ defined by $F_{X}(x)=$ $\{U \in D(X): x \in U\}$ is an order-isomorphism and a homeomorphism. The set of closed increasing (closed decreasing) subsets of a Priestley space $X$ will be denoted by $\mathscr{C}_{i}(X)$ $\left(\mathscr{C}_{d}(X)\right)$. The set of open subsets of a Priestley space $X$ will be denoted by $O(X)$. The set of closed and open (clopen) subsets of $X$ will be denoted by $\operatorname{Clop}(X)$.

Let $X$ be a Priestley space and let $Y$ be a subset of $X$. The closure of $Y$ will be denoted by $\mathrm{Cl}(Y)$. The set of maximal (minimal) elements of $Y$ will be denoted by $\max Y(\min Y)$. Let us recall that for any nonempty closed subset $Y$ of $X, \max Y \neq \varnothing(\min Y \neq \varnothing)$.

Let $\mathbf{A}$ be a bounded distributive lattice and let $Y$ be a closed subset of $X(\mathbf{A})$. It is known that

$$
\theta(Y)=\left\{(a, b) \in A \times A: \sigma_{\mathbf{A}}(a) \cap Y=\sigma_{\mathbf{A}}(b) \cap Y\right\}
$$

is a lattice congruence on $\mathbf{A}$, and the correspondence $Y \rightarrow \theta(Y)$ establishes an antiisomorphism from the lattice of closed subsets of $X(\mathbf{A})$ onto the lattice of lattice of congruences of $\mathbf{A}$ (see [8]). The filter (ideal) generated by a subset $H \subseteq A$ will be denoted by $[H)((H])$. The lattice of all filters (ideals) of $\mathbf{A}$ is denoted by $\operatorname{Fi}(\mathbf{A})(\operatorname{Id}(\mathbf{A}))$.

Let $Y$ be a subset of a set $X$. The theoretical complement of $Y$ is denoted by $Y^{c}=$ $X-Y$.

Definition 2.1. An algebra $\mathbf{A}=\langle A, \vee, \wedge, \square, \diamond, 0,1\rangle$ is a $\square \diamond$-lattice, if $\langle A, \vee, \wedge, 0,1\rangle$ is a bounded distributive lattice and $\square$ and $\diamond$ are unary operations defined on $A$ such that for all $a, b \in A$,

(M1) $\square(a \wedge b)=\square a \wedge \square b$ and $\square 1=1$;

(M2) $\diamond(a \vee b)=\diamond a \vee \diamond b$ and $\diamond 0=0$.

A $\square$-lattice $(\diamond$-lattice) is a bounded distributive lattice $\langle A, \vee, \wedge, 0,1\rangle$ with an operator $\square(\diamond)$ satisfying the condition (M1) (or (M2)).

We will proceed with the duality theory for $\square \diamond$-lattices. Let $R$ be a binary relation on a set $X$. For each $x \in X$, let us consider the subset $R(x)=\{y \in X:(x, y) \in R\}$. For each $U \subseteq X$ define the sets

$$
\begin{aligned}
& \square_{R}(U)=\{x \in X: R(x) \subseteq U\}, \\
& \triangleright_{R}(U)=\{x \in X: R(x) \cap U \neq \varnothing\} .
\end{aligned}
$$

Definition 2.2. A relational Priestley space $[3,11,12,18]$ is a relational structure $\langle X, \leq$, $\left.R_{\square}, R_{\diamond}\right\rangle$, where $\langle X, \leq\rangle$ is a Priestley space, and $R_{\square}$ and $R_{\diamond}$ are binary relations defined on 
$4 \quad$ Simple and subdirectly irreducibles bounded

$X$ such that

(1) $R_{\square}(x) \in \mathscr{C}_{i}(X), R_{\diamond}(x) \in \mathscr{C}_{d}(X)$, for all $x \in X$,

(2) $\square_{R_{\square}}(U), \diamond_{R_{\diamond}}(U) \in D(X)$, for each $U \in D(X)$.

Let $\mathbf{A}$ be a $\square \diamond$-lattice. We define binary relations $R_{\square}^{\mathrm{A}}$ and $R_{\diamond}^{\mathrm{A}}$ on $X(\mathbf{A})$ in the following way:

(1) $(x, y) \in R_{\square}^{\mathrm{A}} \Leftrightarrow \square^{-1}(x) \subseteq y$,

(2) $(x, y) \in R_{\diamond}^{\mathrm{A}} \Leftrightarrow y \subseteq \diamond^{-1}(x)$,

with $x, y \in X(\mathbf{A})$. We will also consider the relation $R_{\mathbf{A}}=R_{\square}^{\mathbf{A}} \cap R_{\diamond}^{\mathbf{A}}$.

For the proof of the following result, see $[3,7,12,16,18]$.

Lemma 2.3. Let $\mathbf{A}$ be $a \square \diamond$-lattice. Then for each $x \in X(\mathbf{A})$ and for each $a \in A$,

(1) $\square a \in x$ if and only if for every $y \in X(\mathbf{A})$ such that $(x, y) \in R_{\square}^{\mathbf{A}}$ it holds $a \in y$;

(2) $\diamond a \in x$ if and only if there exists $y \in X(\mathbf{A})$ such that $(x, y) \in R_{\diamond}^{\mathrm{A}}$ and $a \in y$.

Theorem 2.4. Let $\mathbf{A}$ be $a \square \diamond$-lattice, then the structure $\left\langle X(\mathbf{A}), \subseteq, R_{\square}^{\mathbf{A}}, R_{\diamond}^{\mathbf{A}}\right\rangle$ is a relational Priestley space such that the mapping $\sigma_{\mathrm{A}}: \mathbf{A} \rightarrow D(X(\mathbf{A}))$ is an isomorphism of $\square \diamond$-lattices, that is, $\sigma_{\mathbf{A}}(\square a)=\square_{R}\left(\sigma_{\mathbf{A}}(a)\right)$ and $\sigma_{\mathbf{A}}(\diamond a)=\diamond_{R}\left(\sigma_{\mathbf{A}}(a)\right)$, for all $a \in A$.

Positive modal algebras are $\square \diamond$-lattices where the operators $\square$ and $\diamond$ are connected by two rather weak inequalities (see $[6,7,10,11]$ ).

Definition 2.5. A $\square \diamond$-lattice A is a positive modal algebra, or PM-algebra, if the following conditions are satisfied:

(1) $\square a \wedge \diamond b \leq \diamond(a \wedge b)$,

(2) $\square(a \vee b) \leq \square a \vee \diamond b$, for all $a, b \in A$.

The dual space of a PM-algebra can be defined as a relational Priestley space $\langle X, \leq$, $\left.R_{\square}, R_{\diamond}\right\rangle$ verifying additional conditions. These conditions appear in [11] without detailed proofs. We will provide them for the sake completeness.

Theorem 2.6. Let $\mathbf{A}$ be $\square \diamond$-lattice. Then,

(1) $\square a \wedge \diamond b \leq \diamond(a \wedge b)$ is valid in $\mathbf{A}$ if and only if $R_{\diamond}^{\mathbf{A}} \subseteq\left(R_{\square}^{\mathbf{A}} \cap R_{\diamond}^{\mathbf{A}}\right) \circ \subseteq^{-1}$;

(2) $\square(a \vee b) \leq \square a \vee \diamond b$ is valid in $\mathbf{A}$ if and only if $R_{\square}^{\mathbf{A}} \subseteq\left(R_{\square}^{\mathbf{A}} \cap R_{\diamond}^{\mathbf{A}}\right) \circ \subseteq$.

Proof. We prove only (1). The proof of (2) is similar and left to the reader.

$(\Rightarrow)$ Assume that $\square a \wedge \diamond b \leq \diamond(a \wedge b)$ is valid in $\mathbf{A}$ and let $x, y \in X(\mathbf{A})$ such that $(x, y) \in R_{\diamond}^{\mathrm{A}}$. Let us consider the filter $F=\left[\square^{-1}(x) \cup y\right)$. Then $F \cap \diamond^{-1}(x)^{c}=\varnothing$, because in opposite case we have $p \in \square^{-1}(x), q \in y$, and $d \in \nabla^{-1}(x)^{c}$ such that $p \wedge q \leq d$. So, $\square p \wedge \diamond q \leq \diamond d$, and since $\square p \wedge \diamond q \in x, \diamond d \in x$, which is a contradiction. Thus there exists $z \in X(\mathbf{A})$ such that $(x, z) \in R_{\square}^{\mathrm{A}} \cap R_{\diamond}^{\mathrm{A}}$ and $y \subseteq z$.

$(\Leftarrow)$ Suppose that there exist $a, b \in A$ such that $\square a \wedge \diamond b \not \leq \diamond(a \wedge b)$. Then there exists $x \in X(\mathbf{A})$ such that $\square a, \diamond b \in x$ and $\diamond(a \wedge b) \notin x$. Since $\diamond b \in x$, there exists $y \in X(\mathbf{A})$ such that $(x, y) \in R_{\diamond}^{\mathbf{A}}$ and $b \in y$. By assumption, there exists $z \in X(\mathbf{A})$ such that $(x, z) \in$ $R_{\square}^{\mathrm{A}} \cap R_{\diamond}^{\mathrm{A}}$ and $y \subseteq z$. But as $\square a \in x, a \wedge b \in z$, and this implies that $\diamond(a \wedge b) \in x$, which is a contradiction. Thus $\square a \wedge \diamond b \leq \diamond(a \wedge b)$ is valid in A. 
Remark 2.7. Let $\mathbf{A}$ be a PM-algebra. Let us consider the relation $R_{\mathbf{A}}=R_{\square}^{\mathbf{A}} \cap R_{\diamond}^{\mathbf{A}}$. From Theorem 2.6 it follows that

$$
\begin{gathered}
R_{\square}^{\mathrm{A}}=R_{\mathbf{A}} \circ \subseteq, \\
R_{\diamond}^{\mathrm{A}}=R_{\mathbf{A}} \circ \subseteq^{-1}, \\
\left(\subseteq \circ R_{\mathbf{A}}\right) \subseteq\left(R_{\mathbf{A}} \circ \subseteq\right), \\
\left(\subseteq^{-1} \circ R_{\mathbf{A}}\right) \subseteq\left(R_{\mathbf{A}} \circ \subseteq^{-1}\right) .
\end{gathered}
$$

So we can deduce that the axioms that relate the operators $\square$ and $\diamond$ allow us to simplify the dual space of a positive modal algebra by considering the single relation $R_{\mathrm{A}}=R_{\square}^{\mathrm{A}} \cap$ $R_{\diamond}^{\mathrm{A}}$. Thus, the associated relational Priestley spaces of PM-algebras (called PM-spaces) (see [7]) can be defined as triples $\langle X, \leq, R\rangle$, where $\langle X, \leq\rangle$ is a Priestley space and $R$ is a binary relation on $X$ such that

(1) for each $x \in X, R(x)$ is closed subset of $X$;

(2) for each $x \in X, R(x)=R_{\square}(x) \cap R_{\diamond}(x)$, where $R_{\square}=R \circ \leq$ and $R_{\diamond}=R_{\circ} \leq^{-1}$;

(3) for each $U \in D(X), \square_{R}(U), \diamond_{R}(U) \in D(X)$.

We note that in a PM-space $\langle X, \leq, R\rangle$,

$$
\square_{R}(U)=\square_{R_{\square}}(U), \quad \diamond_{R}(U)=\diamond_{R_{\diamond}}(U),
$$

for every $U \in D(X)$ (for more details on the duality for PM-algebras, see [7]).

Let $\mathbf{A}$ be a $\square \diamond$-lattice. Let $a \in A$. For each $n \geq 0$ we define inductively the formula $\square^{n} a$ as $\square^{0} a=a$ and $\square^{n+1} a=\square \square^{n} a$. The formula $\diamond^{n} a$ is defined similarly. We also define the formulas $\alpha_{n}(a)$ and $\beta_{n}(a)$ as $\alpha_{n}(a)=a \wedge \square a \wedge \cdots \wedge \square^{n} a$ and $\beta_{n}(a)=a \vee \diamond a \vee \cdots \vee$ $\diamond^{n} a$.

For a binary relation $R$ on a set $X$, let $R^{n}$ be the binary relation on $X$ defined inductively by $R^{0}=\operatorname{Id}_{X}$ and $R^{n+1}=R^{n} \circ R$, where $\operatorname{Id}_{X}$ is the identity relation on $X$. The closure reflexive and transitive of $R$ is the relation $R^{*}=\bigcup_{n \geq 0} R^{n}$. We note that if $R$ is reflexive and transitive, then $R^{*}(x)=R(x)$, for each $x \in X$.

\section{Congruences and subdirectly irreducible algebras}

One of the major contributions of the Priestley duality is that it allows us to give an exact characterization of the lattice congruence of a bounded distributive lattice. This characterization has been applied to many classes of algebra, like $p$-algebras, double $p$ algebras, De Morgan algebras, J-distributive lattices [16], and others. In this section it will be shown that the techniques given in [16] to determine the congruences and the simple and subdirectly irreducible algebras in the variety of $J$-lattices (or $\diamond$-lattices in our notation) can also be applied to $\square \diamond$-lattices and PM-algebras. First, we will characterize the closed subsets of a relational Priestley space that correspond to modal congruences, that is, lattice congruences preserving the modal operators. 
Let $\left\langle X, \leq, R_{\square}, R_{\diamond}\right\rangle$ be a relational Priestley space. Let $M(x)=\min R_{\square}(x) \cup \max R_{\diamond}(x)$, for each $x \in X$.

Definition 3.1. Let $\left\langle X, \leq, R_{\square}, R_{\diamond}\right\rangle$ be a relational Priestley space. Let $Y$ be a closed subset of $X$. Say that $Y$ is

(1) $R_{\diamond}$-saturated, if $\max R_{\diamond}(x) \subseteq Y$, for each $x \in Y$;

(2) $R_{\square}$-saturated, if $\min R_{\square}(x) \subseteq Y$, for each $x \in Y$;

(3) $R$-saturated, if $M(x) \subseteq Y$, for each $x \in Y$.

We will denote by $C_{R_{\diamond}}(X), C_{R_{\square}}(X)$, and $C_{R}(X)$, the lattice of $R_{\diamond}$-saturated subsets of $X$, the lattice of $R_{\square}$-saturated subsets of $X$, and the lattice of $R$-saturated subsets of $X$, respectively.

We note that the notion of $R$-saturated is connected with the notion of closed and order-hereditary (or M-hereditary) introduced in [3].

Let $\mathbf{A}$ be a $\square \diamond$-lattice. A lattice congruence $\theta$ of $\mathbf{A}$ is a $\square$-congruence ( $\diamond$-congruence), if for every $a, b \in A$ such that $(a, b) \in \theta$, then $(\square a, \square b) \in \theta((\diamond a, \diamond b) \in \theta)$. A congruence is a lattice congruence $\theta$, that is, a $\square$-congruence and a $\diamond$-congruence. We denote by $\operatorname{Con}(\mathbf{A}, \square)(\operatorname{Con}(\mathbf{A}, \diamond))$ the lattice of all $\square$-congruences $(\diamond$-congruences), and by $\operatorname{Con}(\mathbf{A})$ the lattice of all congruences.

If $\mathbf{A}$ is a lattice, we denote by $\mathbf{A}^{d}$ the lattice with the dual order.

The following result is necessary to determine the simple and subdirectly irreducible PM-algebras.

Lemma 3.2. Let $\mathbf{A}$ be $a \square \diamond$-lattice. Consider the relation $R_{\mathbf{A}}=R_{\square}^{\mathbf{A}} \cap R_{\diamond}^{\mathbf{A}}$. Then

(1) if A satisfies $\square a \wedge \diamond b \leq \diamond(a \wedge b)$, then $\max R_{\diamond}^{\mathrm{A}}(x)=\max R_{\mathbf{A}}(x)$, for all $x \in X(\mathbf{A})$;

(2) if A satisfies $\square(a \vee b) \leq \square a \vee \diamond b$, then $\min R_{\square}^{\mathbf{A}}(x)=\min R_{\mathbf{A}}(x)$, for all $x \in X(\mathbf{A})$.

Proof. It is immediate.

Theorem 3.3. Let $\mathbf{A}$ be a $\square \diamond$-lattice. Then the correspondence $Y \rightarrow \theta(Y)$ establishes the following isomorphisms:

(1) $C_{R_{\square}}(X(\mathbf{A})) \cong \operatorname{Con}(\mathbf{A}, \square)^{d}$;

(2) $C_{R_{\diamond}}(X(\mathbf{A})) \cong \operatorname{Con}((\mathbf{A}, \diamond))^{d}$;

(3) $C_{R}(X(\mathbf{A})) \cong \operatorname{Con}(\mathbf{A})^{d}$.

Proof. We give only a proof the assertion (1) (different from the proof given in [3]). For the proof of assertion (2), see [16] or [3]. Assertion (3) follows by items (1), (2) and Lemma 3.2.

(1) Let $\theta \in \operatorname{Con}(\mathbf{A}, \square)$ and let $Y$ be the closed subset associated with $\theta$. Let $x \in Y$ and $y \in \min R_{\square}^{\mathbf{A}}(x)$. Assume that $y \notin Y$. Since $Y$ is closed, there exist $a, b \in A$ such that $(a \wedge b, a) \in \theta(Y), a \in y$, and $b \notin y$. We prove that

$$
x \cap \square\left(y^{c} \cup\{a\}\right] \neq \varnothing .
$$

Suppose the contrary. Since $y^{c} \cup\{a\}$ is closed under $\vee$, there exists $z \in X(\mathbf{A})$ such that $\square^{-1}(x) \subseteq z$ and $z \subseteq y$. As $y$ is minimal in $R_{\square}^{\mathbf{A}}(x)$, we get $z=y$. It follows that $a \in z$, 
which is a contradiction. Thus there exists some $q \notin y$, such that $\square(q \vee a) \in x$. Since $(a \wedge b, a) \in \theta(Y)$

$$
(\square(q \vee(a \wedge b)), \square(q \vee a)) \in \theta
$$

It follows that $\square(q \vee(a \wedge b)) \in x$, and this implies that $a \wedge b \leq b \in y$, which is a contradiction. Thus $y \in Y$ and $Y \in C_{R_{\square}}(X(\mathbf{A}))$.

Suppose that $Y \in C_{R \square}(X(\mathbf{A}))$. We prove that $\theta(Y)$ preserves the operation $\square$. Let $a, b \in$ $A$ such that $(a, b) \in \theta(Y)$. Suppose that

$$
x \in \sigma_{\mathrm{A}}(\square a) \cap Y, \quad \square b \notin x,
$$

for some $x \in X(\mathbf{A})$. Then from Lemma 2.3 there exists $y \in R_{\square}^{\mathrm{A}}(x)$ such that $b \notin y$. Since $R_{\square}^{\mathrm{A}}(x)$ is a closed subset of $X(\mathbf{A})$, there exists $z \in X(\mathbf{A})$ such that $z \in \min R_{\square}^{\mathrm{A}}(x)$ and $z \subseteq y$ (for a proof of this fact see [3, Proposition 4.10]). It follows that $z \in Y$. Then $z \in \sigma_{\mathbf{A}}(a) \cap$ $Y=\sigma_{\mathbf{A}}(b) \cap Y$, that is, $b \in z$, which is a contradiction. Thus $\theta(Y)$ is a $\square$-congruence.

Lemma 3.4. Let $\left\langle X, \leq, R_{\square}, R_{\diamond}\right\rangle$ be a relational Priestley space. Then the following conditions are mutually exclusive:

(1) $\left\{x \in X: \mathrm{Cl}_{R}(M(x))=X\right\} \in \mathrm{O}(X)-\{\varnothing, X\}$;

(2) there exists $x \in X$ with $x \notin \mathrm{Cl}_{R}(M(x))$ such that $\{x\} \cup \mathrm{Cl}_{R}(M(x))=X$ and $R_{\square}(x) \cup$ $R_{\diamond}(x) \neq \varnothing$.

Proof. Suppose that the conditions (1) and (2) are not mutually exclusive. Then there are $x, y$ in $X$ such that

$$
\begin{array}{cl}
\mathrm{Cl}_{R}(M(x))=X, \quad & y \notin \mathrm{Cl}_{R}(M(y)), \quad\{y\} \cup \mathrm{Cl}_{R}(M(y))=X, \\
& R_{\square}(y) \cup R_{\diamond}(y) \neq \varnothing .
\end{array}
$$

We note that $\mathrm{Cl}_{R}(M(y)) \neq \varnothing$, because $R_{\square}(y) \cup R_{\diamond}(y) \neq \varnothing$. It is clear that $x \neq y$, and thus $x \in \mathrm{Cl}_{R}(M(y))$. As $\mathrm{Cl}_{R}(M(y))$ is $R$-saturated we have that $M(x) \subseteq \mathrm{Cl}_{R}(M(y))$, which is a contradiction since by hypothesis $\mathrm{Cl}_{R}(M(x))=X$ and besides $\mathrm{Cl}_{R}(M(y)) \neq X$.

The proof of the following result is established for $\square \diamond$-lattices and follows the proof for the characterization of simple and subdirectly irreducible algebras in the variety of $\diamond$-lattices given in [16]. It is easy to formulate a similar result for $\square$-lattices, and PMalgebras (using Lemma 3.2). Note that the following characterization is more complete than the results obtained in [3], because Birchall only gives a sufficient condition for a $\square \diamond$-lattice A is subdirectly irreducible.

Theorem 3.5. Let $\left\langle X, \leq, R_{\square}, R_{\diamond}\right\rangle$ be the relational Priestley space of a $\square \diamond$-lattice $\mathbf{A}$. Then

(1) $\mathbf{A}$ is simple if and only if either for all $x \in X, R_{\square}(x) \cup R_{\diamond}(x) \neq \varnothing$, and $\mathrm{Cl}_{R}(M(x))=$ $X$, or for all $x \in X, R_{\square}(x) \cup R_{\diamond}(x)=\varnothing$, and $X$ is a singleton;

(2) A is subdirectly irreducible but nonsimple if and only if one and only one of the following conditions holds true:

(a) $\left\{x \in X: \mathrm{Cl}_{R}(M(x))=X\right\} \in \mathcal{O}(X)-\{\varnothing, X\}$;

(b) there exists $x \notin \mathrm{Cl}_{R}(M(x))$ such that $\{x\} \cup \mathrm{Cl}_{R}(M(x))=X$ and $R_{\square}(x) \cup R_{\diamond}(x)$ $\neq \varnothing$. 
Proof. (1) ( $\Rightarrow$ ). Let A be a simple $\square \diamond$-lattice. Suppose that for all $x \in X, R_{\square}(x) \cup R_{\diamond}(x) \neq$ $\varnothing$. Let $x \in X$. Therefore $R_{\square}(x) \neq \varnothing$ or $R_{\diamond}(x) \neq \varnothing$. This implies that $\min R_{\square}(x) \neq \varnothing$ or $\max R_{\diamond}(x) \neq \varnothing$. So

$$
\mathrm{Cl}_{R}(M(x))=X
$$

because $\mathbf{A}$ is simple.

Now we suppose that $x \in X$ and $R_{\square}(x) \cup R_{\diamond}(x)=\varnothing$. Then $R_{\square}(x)=\varnothing$ and $R_{\diamond}(x)=$ $\varnothing$. So $\{x\}$ is an $R$-saturated subset of $X$. Since $\mathbf{A}$ is simple, we have $\{x\}=X$ and consequently $X$ is a singleton.

We prove the other implication. Let us assume that $R_{\square}(x) \cup R_{\diamond}(x) \neq \varnothing$ and $\mathrm{Cl}_{R}(M(x))$ $=X$, for every $x \in X$. Let $Y$ be a nonempty $R$-saturated subset of $X$. Then, for every $x \in Y$, $M(x) \subseteq Y$. So $\mathrm{Cl}_{R}(M(x)) \subseteq Y$. Therefore $Y=X$, and consequently $\mathbf{A}$ is simple.

If $R_{\square}(x) \cup R_{\diamond}(x)=\varnothing$, for every $x \in X$, and $X$ is a singleton, it is clear that the sets $\varnothing$ and $X$ are the only $R$-saturated subsets, and thus $\mathbf{A}$ is simple.

(2) From Lemma 3.4 it follows that the conditions (a) and (b) are mutually exclusive.

Suppose that $\mathbf{A}$ is subdirectly irreducible but not simple. Let $Y$ be the greatest element of $C_{R}(X)-\{X\}$. Since $\mathbf{A}$ is not simple, $Y \neq \varnothing$. We define the set

$$
T=\left\{x \in X: \mathrm{Cl}_{R}(M(x)) \neq(X)\right\}
$$

Since $Y$ is $R$-saturated and different from $X$ we have $Y \subseteq T$. Suppose that $Y=T$. Clearly $X-Y$ is a nonempty open subset of $X$, and in accordance with the definition of $T$, we obtain (a).

Now, suppose that $Y \subsetneq T$. Let $x \in T-Y$. Then $\mathrm{Cl}_{R}(M(x))$ is an $R$-saturated subset of $X$, and different from $X$. Thus $\mathrm{Cl}_{R}(M(x)) \subseteq Y$. It is clear that the set $\{x\} \cup \mathrm{Cl}_{R}(M(x))$ is an $R$-saturated subset of $X$. Since $x \notin Y$, we may conclude that

$$
\{x\} \cup \mathrm{Cl}_{R}(M(x))=X
$$

and thus $Y=\mathrm{Cl}_{R}(M(x))$. So, we obtain (b).

Let us prove the reciprocal. Suppose (a). Let $T$ be the set defined previously. We can see that $T$ is different from $X$, because otherwise the set $\left\{x \in X: \mathrm{Cl}_{R}(M(x))=X\right\}$ would be empty, again by the hypothesis (a). Also it is clear that $\mathbf{A}$ is not simple, and $T$ is closed.

We show that $T$ is $R$-saturated. Let $x \in T$ and suppose $z \in M(x)$. So $z \in \mathrm{Cl}_{R}(M(x))$ and consequently $M(z)$ is $R$-saturated. Therefore $T \in C_{R}(X)$.

We see now that $T$ is the greatest element of $C_{R}(X)-\{X\}$. Let $Y \in C_{R}(X)-\{X\}$. Let $x \in Y$. Then $M(x) \subseteq Y$ and thus $\mathrm{Cl}_{R}(M(x)) \subseteq Y$. Since $Y \neq X$, we have that $x \in T$, that is, $Y \subseteq T$.

Now suppose (b). It is clear that $\mathbf{A}$ is not simple. We consider the set

$$
Z=\mathrm{Cl}_{R}(M(x))
$$


Since $R_{\square}(x) \cup R_{\diamond}(x) \neq \varnothing, Z$ is a nonempty $R$-saturated set different from $X$, where $x$ is the element considered in (b). Let $Y \in C_{R}(X)-\{X\}$ and let $y \in Y$. If $y=x$, since $Y \in C_{R}(X)$, we have $X=\{x\} \cup \mathrm{Cl}_{R}(M(x)) \subseteq Y$, which is a contradiction. So, $y \neq x$ and $y \in \mathrm{Cl}_{R}(M(x))=Z$. Thus, $Y \subseteq Z$.

\section{Topological and monadic positive modal algebras}

In this section we will introduce the variety of topological positive modal algebras and the variety of monadic positive modal algebras, and we will determine the simple and subdirectly irreducible algebras in these varieties.

Let $\mathbf{A}$ be a $\square \diamond$-lattice. We will write $\mathbf{A} \vDash \alpha \leq \beta$ when the inequality $\alpha \leq \beta$ is valid in A. In the next result we will establish that certain additional conditions defined in a $\square \diamond$ lattice correspond to additional properties defined in the dual space. The proof of these correspondences can be deduced from the results established in [6]. Another method where these results can be deduced is taking into account that all these conditions are Sahlqvist equations, and thus the proof also follows by the general results given in [11].

Theorem 4.1. Let $\mathbf{A}$ be $a \square \diamond$-lattice. Let $R_{\square}=R_{\square}^{\mathrm{A}}$ and let $R_{\diamond}=R_{\diamond}^{\mathrm{A}}$. Then

(1) $\mathbf{A} \vDash \square a \leq a$ if and only if $R_{\square}$ is reflexive;

(2) $\mathbf{A} \vDash a \leq \diamond a$ if and only if $R_{\diamond}$ is reflexive;

(3) $\mathbf{A} \vDash \square a \leq \square^{2} a$ if and only if $R_{\square}$ is transitive;

(4) $\mathbf{A} \vDash \diamond^{2} a \leq \diamond a$ if and only if $R_{\diamond}$ is transitive;

(5) $\mathbf{A} \vDash a \leq \square \diamond a$ if and only if $R_{\square} \subseteq\left(R_{\diamond}\right)^{-1}$;

(6) $\mathbf{A} \vDash \diamond \square a \leq a$ if and only if $\left(R_{\diamond}\right)^{-1} \subseteq R_{\square}$;

(7) $\mathbf{A} \vDash \alpha_{n}(a) \leq \square^{n+1} a$ if and only if $\left(R_{\square}\right)^{n+1}(x) \subseteq[x) \cup R_{\square}(x) \cdots \cup\left(R_{\square}\right)^{n}(x)$ for every $x \in X(\mathbf{A})$.

Definition 4.2. Let A be a PM-algebra. Say that A is a topological PM-algebra, or TPMalgebra, if A satisfies the following axioms:

(1) $\square a \leq a, a \leq \diamond a$;

(2) $\square a \leq \square^{2} a, \diamond^{2} a \leq \diamond a$, for all $a \in A$.

Remark 4.3. By Remark 2.7 and Theorem 4.1 we have that the relational Priestley space of a TPM-algebra $\mathbf{A}$ is the PM-space $\left\langle X(\mathbf{A}), \subseteq, R_{\mathbf{A}}\right\rangle$, where $R_{\mathbf{A}}=R_{\square}^{\mathbf{A}} \cap R_{\diamond}^{\mathbf{A}}$ is a reflexive and transitive relation. The space associated with a TPM-algebra will be called a TPM-space.

Remark 4.4. We note that in a TPM-space $\langle X, \leq, R\rangle, R^{*}(x)=R(x)$, for each $x \in X$. Moreover, taking into account that $\min R_{\square}(x)=\min R(x) \subseteq R(x), \max R_{\diamond}(x)=\max R(x) \subseteq$ $R(x)$, and that $R(x)$ is a closed subset of $X$, we have that $R(x)$ is an $R$-saturated subset, for each $x \in X$. By these facts it is easy to see that the following conditions are equivalent for each $x \in X$,

(1) $\mathrm{Cl}_{R}(M(x))=X$;

(2) $R(x)=X$ and $\mathrm{Cl}_{R}(\min X \cup \max X)=X$;

(3) $R_{\square}(x)=X$, and $R_{\diamond}(x)=X$ and $\mathrm{Cl}_{R}(\min X \cup \max X)=X$.

We need the following auxiliary result to give the characterization of simple and subdirectly irreducible TPM-algebras. 
Proposition 4.5. Let $\left\langle X, \leq, R_{\square}, R_{\diamond}\right\rangle$ be a relational Priestley space. Then

(1) $\left\{x \in X: R_{\square}(x)=X\right\}=\bigcap\left\{\left(\square_{R_{\square}}(U)\right)^{c}: U \in D(X)-\{X\}\right\}$;

(2) $\left\{x \in X: R_{\diamond}(x)=X\right\}=\bigcap\left\{\diamond_{R_{\diamond}}(U): U \in D(X)-\{\varnothing\}\right\}$.

Proof. We prove only (1). The proof of (2) is similar.

(1) Let $x \in X$ such that $R_{\square}(x)=X$. Suppose that there exists $U \in D(X)-\{X\}$ such that $x \in \square_{R_{\square}}(U)$. Then $R_{\square}(x) \subseteq U$, and thus $U=X$, which is a contradiction. Therefore $x \notin \square_{R_{\square}}(U)$ for all $U \in D(X)-\{X\}$.

Let $x \in X$. If $R_{\square}(x) \neq X$, then there exists $y \in X$ such that $y \notin R \square(x)$. Since $R_{\square}(x)$ is an increasing and closed subset of $X$, there is $U \in D(X)-\{X\}$ such that $y \notin U$ and $R_{\square}(x) \subseteq U$. Thus $x \notin \bigcap\left\{\left(\square_{R_{\square}}(U)\right)^{c}: U \in D(X)-\{X\}\right\}$.

Lemma 4.6. Let $\left\langle X, \leq, R_{\square}, R_{\diamond}\right\rangle$ be a TPM-space. The following conditions are equivalent:

(1) $S=\left\{x \in X: \mathrm{Cl}_{R}(M(x))=X\right\} \in O(X)-\{\varnothing, X\}$;

(2) $\mathrm{Cl}_{R}(\min X \cup \max X)=X$, and $H=\{x \in X: R(x)=X\} \in \operatorname{Clop}(X)-\{\varnothing, X\}$.

Proof. $(\Rightarrow)$ Let $x \in H$. As $R(x)=X$,

$$
\mathrm{Cl}_{R}(M(x))=\mathrm{Cl}_{R}(\min X \cup \max X)=X .
$$

From Remark 4.4 we get $x \in S$. As $S \in \mathcal{O}(X)-\{\varnothing, X\}$, there exists $O_{x} \in \mathcal{O}(X)-\{\varnothing, X\}$ such that $x \in O_{x} \subseteq S$. Clearly $O_{x} \subseteq H$. Thus, $H \in \mathcal{O}(X)-\{\varnothing, X\}$. On the other hand, $H=H_{\square} \cap H_{\diamond}$, where $H_{\square}=\left\{x \in X: R_{\square}(x)=X\right\}$ and $H_{\diamond}=\left\{x \in X: R_{\diamond}(x)=X\right\}$. From Proposition 4.5 it follows that $H_{\square}$ and $H_{\diamond}$ are closed subsets of $X$. Thus $H \in \operatorname{Clop}(X)-$ $\{\varnothing, X\}$.

$(\Leftarrow)$ It follows from Remark 4.4.

Theorem 4.7. Let A be a TPM-algebra. Let $\langle X, \leq, R\rangle$ be the TPM-space associated. Then,

(1) $\mathbf{A}$ is simple if and only if $\mathrm{Cl}_{R}(\min X \cup \max X)=X$, and $R(x)=X$, for each $x \in X$;

(2) A is subdirectly irreducible but nonsimple if and only if one and only one of the following conditions are valid:

(a) $\mathrm{Cl}_{R}(\min X \cup \max X)=X$, and $\{x \in X: R(x)=X\} \in \operatorname{Clop}(X)-\{\varnothing, X\}$;

(b) there exists $x \in X$ such that $R(x)=X, x \notin \mathrm{Cl}_{R}(\max X \cup \min X)$, and $\{x\} \cup$ $\mathrm{Cl}_{R}(\max X \cup \min X)=X$.

Proof. Assertion (1) follows by Theorem 3.5 and Remark 4.4 taking into account that $R(x)=R_{\square}(x) \cap R_{\diamond}(x), R_{\square}(x) \neq \varnothing$, and $R_{\diamond}(x) \neq \varnothing$, for each $x \in X$, because $R_{\square}$ and $R_{\diamond}$ are reflexive.

Assertion (2) follows by Theorem 3.5, Remark 4.4, and Lemma 4.6.

Definition 4.8. Let $\mathbf{A}$ be a PM-algebra. Say that $\mathbf{A}$ is a monadic positive modal algebra, or MPM-algebra, if $\mathbf{A}$ is a TPM-algebra such that

(S1) $a \leq \square \diamond a$;

(S2) $\diamond \square a \leq a$, for every $a \in A$.

From the results given in [7] or [11] we have that a PM-algebra A is an MPM-algebra if and only if the binary relations $R_{\square}^{\mathrm{A}}$ and $R_{\diamond}^{\mathrm{A}}$ are equivalence relations if and only if the relation $R_{\mathrm{A}}=R_{\square}^{\mathrm{A}} \cap R_{\diamond}^{\mathrm{A}}$ is an equivalence. 
LEMmA 4.9. If $\mathbf{A}$ is an MPM-algebra. Then the following conditions hold:

(1) $\diamond \square a=\square a$,

(2) $\square \diamond a=\diamond a$,

(3) $\square(\square a \vee b)=\square a \vee \square b$,

(4) $\diamond(a \wedge \diamond b)=\diamond a \wedge \diamond b$.

Proof. (1) Let $a \in A$. Since $\square a=\square \square a, \diamond \square a=\diamond \square \square a$. By condition (S2) we deduce that $\diamond \square a \leq \square a$. From condition (S1) we have $\square a \leq \diamond \square a$. Thus we conclude that $\diamond \square a=\square a$. The proof of (2) is similar.

(3) Let $a, b \in A$. Since $\square a \vee b \leq \square a \vee b$,

$$
\square(\square a \vee b) \leq \diamond \square a \vee \square b=\square a \vee \square b
$$

On the other hand, as $b \leq \square a \vee b$ and $\square a \leq \square a \vee b, \square b \leq \square(\square a \vee b)$, and $\square \square a=\square a \leq$ $\square(\square a \vee b)$. Thus, $\square a \vee \square b \leq \square(\square a \vee b)$, and consequently the identity (3) is valid.

The proof of (4) is similar and left to the reader.

Lemma 4.10. If $\mathbf{A}$ is an MPM-algebra, then $M(x) \subseteq \min X(\mathbf{A}) \cup \max X(\mathbf{A})$, for each $x \in$ $\min X(\mathbf{A}) \cup \max X(\mathbf{A})$. Thus, $\operatorname{Cl}(\min X(\mathbf{A}) \cup \max X(\mathbf{A}))$ is an $R$-saturated subset of $X(\mathbf{A})$.

Proof. We prove only that if $x \in \min X(\mathbf{A})$ and $y \in \min R_{\square}^{\mathrm{A}}(x)$, then $y \in \min X(\mathbf{A})$. By Lemma 3.2, $\min R_{\square}^{\mathrm{A}}(x)=\min R_{\mathrm{A}}(x)$. We note that

$$
\square^{-1}(x)=\square^{-1}(y)
$$

because if $\square a \in y$, as $y \in R_{\mathbf{A}}(x)$, then $\diamond \square a \in x$, and by the inequality $\diamond \square a \leq \square a, \square a \in x$ follows. Thus $\square^{-1}(y) \subseteq \square^{-1}(x)$. The other inclusion follows by the inequality $\square a \leq \square \square a$.

Now, we prove that for each $d \in y$ there exists $z \notin y$ such that $d \vee z=1$. Let $x \in y$. Then

$$
\square^{-1}(x) \cap\left(y^{c} \cup\{d\}\right] \neq \varnothing,
$$

because in the opposite case there exists $z \in X(\mathbf{A})$ such that $\square^{-1}(x) \subseteq z \subseteq y$ and $d \notin z$. Since $y$ is minimal in $R_{\square}(x), y=z$, and consequently $d \notin y$, which is a contradiction. So there exists $p \in \square^{-1}(x)$ and there exists $q \notin y$ such that $p \leq q \vee d$. Then $\square p \leq \square(q \vee x) \in x$. As $x$ is minimal, there exists $a \notin x$ such that $\square(q \vee d) \vee a=1$. Then

$$
1=\square 1=\square(\square(q \vee d) \vee a) \leq \square(q \vee d) \vee a
$$

Since $a \notin x, \square(q \vee d) \in x$, and as $\square^{-1}(x)=\square^{-1}(y), d \vee q \in y$. Thus for each $d \in y$ there exists $q \vee a \notin y$ such that $x \vee q \vee a=1$. Then $y$ is a minimal prime filter.

Definition 4.11. Let A be an MPM-algebra. Say that the pair $\{\square, \diamond\}$ is simple if and only if $\square a=0$ for all $a \in A-\{1\}$, and $\nabla a=1$ for all $a \in A-\{0\}$. 


\section{Simple and subdirectly irreducibles bounded}

\section{Lemma 4.12. Let A be an MPM-algebra. Then}

(1) the pair $\{\square, \diamond\}$ is simple if and only if $R_{\mathbf{A}}(x)=X(\mathbf{A})$, for each $x \in X(\mathbf{A})$;

(2) if $\mathbf{A}$ is subdirectly irreducible, then the pair $\{\square, \diamond\}$ is simple;

(3) if $\mathbf{A}$ is subdirectly irreducible, and $Y$ is a proper $R$-saturated subset of $X(\mathbf{A})$, then $\min X(\mathbf{A}) \cup \max X(\mathbf{A}) \subseteq Y$.

Proof. (1) Assume that the pair $\{\square, \diamond\}$ is simple. Suppose that there exists $x \in X(\mathbf{A})$ such that $R_{\mathbf{A}}(x) \neq X(\mathbf{A})$. Since $R_{\mathbf{A}}=R_{\square}^{\mathbf{A}} \cap R_{\diamond}^{\mathbf{A}}$,

$$
R_{\square}^{\mathbf{A}}(x) \neq X(\mathbf{A}) \quad \text { or } \quad R_{\diamond}^{\mathbf{A}}(x) \neq X(\mathbf{A}) .
$$

If $R_{\square}^{\mathbf{A}}(x) \neq X(\mathbf{A})$, then there exists $y \in X(\mathbf{A})$ and there exists $b \in A$ such that $y \notin R_{\square}^{\mathbf{A}}(x)$, $x \in \sigma_{\mathbf{A}}(\square b)$, and $y \notin \sigma_{\mathbf{A}}(b)$. But since $\{\square, \diamond\}$ is simple, we get $\sigma_{\mathbf{A}}(\square b)=\varnothing$, which is a contradiction. The proof of the case $R_{\diamond}^{\mathbf{A}}(x) \neq X(\mathbf{A})$ is similar. Therefore $R_{\mathbf{A}}(x)=X(\mathbf{A})$ for all $x \in X(\mathbf{A})$.

If $R_{\mathbf{A}}(x)=X(\mathbf{A})$ for all $x \in X(\mathbf{A})$, it is easy to check that the pair $\{\square, \diamond\}$ is simple.

(2) Assume that $\mathbf{A}$ is subdirectly irreducible. We prove that $R_{\mathbf{A}}(x)=X(\mathbf{A})$, for each $x \in$ $X(\mathbf{A})$. Suppose that there exists $x \in X(\mathbf{A})$ such that $R_{\mathbf{A}}(x) \neq X(\mathbf{A})$. Since $\mathbf{A}$ is subdirectly irreducible, there exists a greatest proper $R$-saturated subset $Y$ of $X(\mathbf{A})$. So, there exists $y \in X(\mathbf{A})-Y$. Since $R_{\mathbf{A}}$ is an equivalence relation, and the subsets $R_{\mathbf{A}}(x)$ and $R_{\mathbf{A}}(y)$ are $R$-saturated subsets of $X(\mathbf{A})$, we get

$$
R_{\mathbf{A}}(x) \subseteq Y \subset R_{\mathbf{A}}(y)=X(\mathbf{A})
$$

Thus, $x \in R_{\mathbf{A}}(y)$, and this implies that $R_{\mathbf{A}}(x)=R_{\mathbf{A}}(y)$, which is a contradiction. Therefore, $R_{\mathbf{A}}(x)=X(\mathbf{A})$, for each $x \in X(\mathbf{A})$.

(3) Let $\mathbf{A}$ be subdirectly irreducible, and let $Y$ be a proper $R$-saturated subset of $X(\mathbf{A})$. Suppose that there exists $y \in \min X(\mathbf{A})$ such that $y \notin Y$. Then for each $x \in Y, x \varsubsetneqq y$. So for each $x \in Y$ there exists $a_{P} \in A$ such that $a_{P} \in X$ and $a_{P} \notin y$. Then

$$
Y \subseteq \bigcup\left\{\sigma_{\mathbf{A}}\left(a_{x}\right): x \in Y\right\}
$$

As $Y$ is closed, $Y$ is compact. So there exists $a \in A$ such that $Y \subseteq \sigma_{\mathrm{A}}(a)$ and $a \notin y$. Then $(a, 1) \in \theta(Y)$, and consequently $(\square a, \square 1) \in \theta(Y)$. From item (2), the pair $\{\square, \diamond\}$ is simple. So, $(\square a, \square 1)=(0,1) \in \theta(Y)$, that is, $Y=\varnothing$, which is a contradiction. Thus, $\min X(\mathbf{A}) \subseteq Y$. The proof of the inclusion $\max X(\mathbf{A}) \subseteq Y$ is similar.

Proposition 4.13. Let $\mathbf{A}$ be an MPM-algebra. Then,

(1) $\mathbf{A}$ is simple if and only if $\operatorname{Cl}(\min X(\mathbf{A}) \cup \max X(\mathbf{A}))=X(\mathbf{A})$, and the pair $\{\square, \diamond\}$ is simple;

(2) A is subdirectly irreducible but nonsimple if and only if the pair $\{\square, \diamond\}$ is simple and there exists $x \in X(\mathbf{A})$ with $x \notin \mathrm{Cl}(\min X(\mathbf{A}) \cup \max X(\mathbf{A}))$ such that $\{x\} \cup \mathrm{Cl}(\min X(\mathbf{A})$ $\cup \max X(\mathbf{A}))=X(\mathbf{A})$. 
Proof. (1) Since every MPM-algebra is a TPM-algebra, from assertion (1) of Theorem 4.7 and Lemma 4.10, we have the desired result.

(2) that A is subdirectly irreducible but nonsimple. From Lemma 4.12 the pair $\{\square, \diamond\}$ is simple. From Lemma 4.10,

$$
Y=\mathrm{Cl}_{R}(\min X(\mathbf{A}) \cup \max X(\mathbf{A})) \in C_{R}(X(\mathbf{A}))-\{\varnothing\}
$$

and by part $1, Y \neq X(\mathbf{A})$, because $\mathbf{A}$ is not simple.

Let $Z$ be the greatest proper $R$-saturated subset of $X(\mathbf{A})$. Then there exists $x \in X(\mathbf{A})-$ $Z$. We prove that

$$
Y \cup\{x\} \in C_{R}(X(\mathbf{A}))
$$

Let $z \in Y \cup\{x\}$. If $z \in Y$, then $\mathrm{Cl}\left(\min R_{\mathbf{A}}(z) \cup \max R_{\mathbf{A}}(z)\right) \subseteq Y$, because $Y \in C_{R}(X(\mathbf{A}))$. If $z=x$, then from item (3) of Lemma 4.12 and taking into account that the pair $\{\square, \diamond\}$ is simple, we get

$$
\mathrm{Cl}\left(\min R_{\mathbf{A}}(x) \cup \max R_{\mathbf{A}}(x)\right)=\mathrm{Cl}(\min X(\mathbf{A}) \cup \max X(\mathbf{A}))=Y .
$$

Thus $Y \cup\{x\} \in C_{R}(X(\mathbf{A}))$. As $Z$ is a greatest proper $R$-saturated subset of $X(\mathbf{A}), Y \subseteq Z$, and $x \notin Z$, we conclude that $Y \cup\{x\}=X(\mathbf{A})$.

Conversely, suppose that the pair $\{\square, \diamond\}$ is simple and there exists $x \in X(\mathbf{A})$ with $x \notin \mathrm{Cl}(\min X(\mathbf{A}) \cup \max X(\mathbf{A}))$ such that $\{x\} \cup \mathrm{Cl}(\min X(\mathbf{A}) \cup \max X(\mathbf{A}))=X(\mathbf{A})$. From Lemma 4.10, $Y=\mathrm{Cl}_{R}(\min X(\mathbf{A}) \cup \max X(\mathbf{A})) \in C_{R}(X(\mathbf{A}))$. It is clear that if $Z \in C_{R}(X(\mathbf{A}))$ $-\{X(\mathbf{A}), \varnothing\}$, then $Z \subseteq Y$. Thus, $\mathbf{A}$ is subdirectly irreducible.

\section{Heyting modal algebras}

In $[14,19]$ characterizations of subdirectly irreducible algebras for some Heyting algebras with modal operators were given. Similar characterizations for monadic Heyting were given in [2]. In this section we will apply the previous results to give a characterization of the simple and subdirectly irreducible for some Heyting algebras with modal operators in terms of relational Priestley spaces. These characterizations are new (as far as we know). We also note that neither [14] nor [19] gives characterization for simple algebras.

An algebra $\mathbf{A}=\langle A, \vee, \wedge, \rightarrow, 0,1\rangle$ is a Heyting algebra if $\langle A, \vee, \wedge, 0,1\rangle$ is a bounded distributive lattice and $\rightarrow$ is a binary operation such that for all $a, b, c \in A$,

$$
a \wedge b \leq c \quad \text { iff } a \leq b \longrightarrow c .
$$

Definition 5.1. A Priestley space $\langle X, \leq\rangle$ is a Heyting space, or $H$-space, if for every $U, V \in$ $D(X)$, the set

$$
U \Longrightarrow V=\{x \in X:[x) \cap U \subseteq V\} \in D(X) .
$$

It is clear that if $\langle X, \leq\rangle$ is an $H$-space, then $\langle D(X), \cup, \cap, \Rightarrow, \varnothing, X\rangle$ is a Heyting algebra under the implication $\Rightarrow$. 


\section{Simple and subdirectly irreducibles bounded}

Proposition 5.2. Let $\mathbf{A}$ be Heyting algebra. Then $\langle X(\mathbf{A}), \subseteq\rangle$ is an $H$-space such that the map $\sigma_{\mathbf{A}}: \mathbf{A} \rightarrow D(X(\mathbf{A}))$ is a Heyting isomorphism, that is, $\sigma_{\mathbf{A}}$ is a lattice isomorphism such that

$$
\sigma_{\mathbf{A}}(a) \Longrightarrow \sigma_{\mathbf{A}}(b)=\sigma_{\mathbf{A}}(\longrightarrow b)
$$

Let us recall that under the Priestley duality, the lattice of all filters of a bounded distributive lattice is dually isomorphic to the lattice of all increasing closed subsets of the dual space. Under that isomorphism, any filter $F$ of a bounded distributive lattice A corresponds to the increasing closed

$$
Y_{F}=\{x \in X(\mathbf{A}): F \subseteq x\}=\bigcap\left\{\sigma_{\mathbf{A}}(a): a \in F\right\},
$$

and any increasing closed subset $Y$ of $X(\mathbf{A})$ corresponds to the filter

$$
F_{Y}=\left\{a \in A: Y \subseteq \sigma_{\mathrm{A}}(a)\right\}
$$

On the other hand, it is known that there exists a lattice isomorphism between the lattice of all filters and the lattice of all congruences of a Heyting algebra A (see [1]). Under that isomorphism, any congruence $\theta$ corresponds to the filter

$$
F_{\theta}=\{a \in A:(a, 1) \in \theta\}
$$

and any filter $F$ corresponds to the congruence $\theta(F)$ is defined by

$$
(a, b) \in \theta(F) \quad \text { iff }(a \longrightarrow b) \wedge(b \longrightarrow a) \in F .
$$

Thus, there exists an isomorphism from the lattice of congruences of a Heyting algebra $\mathbf{A}$ onto the lattice of increasing closed subsets of the $H$-space $X(\mathbf{A})$.

Definition 5.3. $\mathrm{A} \square$-Heyting algebra is an algebra $\mathbf{A}=\langle A, \vee, \wedge, \rightarrow, \square, 0,1\rangle$ such that $\langle A, \vee$, $\wedge, \rightarrow, 0,1\rangle$ is a Heyting algebra and $\langle A, \vee, \wedge, \square, 0,1\rangle$ is a $\square$-lattice.

A $\diamond$-Heyting algebra is an algebra $\langle A, \vee, \wedge, \rightarrow, \diamond, 0,1\rangle$ such that $\langle A, \vee, \wedge, \rightarrow, 0,1\rangle$ is a Heyting algebra, and $\langle A, \vee, \wedge, \diamond, 0,1\rangle$ is a $\diamond$-lattice.

$\mathrm{A} \square \diamond$-Heyting algebra is an algebra $\langle A, \vee, \wedge, \rightarrow, \square, \diamond, 0,1\rangle$ such that $\langle A, \vee, \wedge, \rightarrow, 0,1\rangle$ is a Heyting algebra, and $\langle A, \vee, \wedge, \square, \diamond, 0,1\rangle$ is a $\square \diamond$-lattice.

A modal Heyting algebra is a $\square \diamond$-Heyting algebra $\langle A, \vee, \wedge, \rightarrow, \square, \diamond, 0,1\rangle$ satisfying the condition $\diamond a \wedge \square b \leq \diamond(a \wedge b)$ for all $a, b \in A$.

We note that the variety of monadic Heyting algebras is a subvariety of modal Heyting algebras, because a monadic Heyting algebra A [2] is a modal Heyting algebra satisfying for all $a, b \in A$ the conditions

$$
\square a \leq a \leq \diamond a, \square \diamond a=\diamond a, \diamond \square a=\square a, \quad \diamond(a \wedge \diamond b)=\diamond a \wedge \diamond b .
$$

We note also that a monadic Heyting algebra is not a monadic positive algebra, since in general the condition $\square(a \vee \square b)=\square a \vee \square b$ is not valid. 
The Priestley duality for $\square$-Heyting algebras, $\diamond$-Heyting algebra, $\square \diamond$-Heyting algebras, and for modal Heyting algebra are consequences of the duality for Heyting algebras and the duality for $\square$-lattices, $\diamond$-lattices, and $\square \diamond$-lattices, respectively. More precisely;

(1) the Priestley space of a $\square$-Heyting ( $\diamond$-algebra) is a triple $\left\langle X, \leq, R_{\square}\right\rangle\left(\left\langle X, \leq, R_{\diamond}\right\rangle\right)$ such that $\langle X, \leq\rangle$ is a $H$-space, $R_{\square}(x) \in \mathscr{C}_{i}(X)\left(R_{\diamond}(x) \in \mathscr{C}_{d}(X)\right)$ every $x \in X$, and $\square_{R_{\square}}(U) \in$ $D(X)\left(\diamond_{R_{\diamond}}(U) \in D(X)\right)$ for every $U \in D(X)$;

(2) the Priestley space of a $\square \diamond$-Heyting algebra is a relational Priestley space $\langle X, \leq$, $\left.R_{\diamond}, R_{\square}\right\rangle$ such that $\langle X, \leq\rangle$ is a $H$-space;

(3) the Priestley space of a modal Heyting algebra is a relational Priestley space $\langle X, \leq$, $\left.R_{\diamond}, R_{\square}\right\rangle$ such that $\langle X, \leq\rangle$ is a $H$-space, and $R_{\diamond} \subseteq\left(R_{\square} \cap R_{\diamond}\right) \circ \subseteq^{-1}$ (by Theorem 2.6).

We will prove that in a modal Heyting algebra any $\square$-congruence is also a $\square \diamond$-congruence.

Lemma 5.4. Let $\mathbf{A}$ be a $\square$-Heyting algebra. Let $Y$ be an increasing closed subset of $X(\mathbf{A})$. Then $\min R_{\square}^{\mathrm{A}}(x) \subseteq Y$ if and only if $R_{\square}^{\mathrm{A}}(x) \subseteq Y$, for each $x \in Y$.

Proof. The direction $(\Rightarrow)$ follows by the fact that $R_{\square}^{A}(x)$ is an increasing subset of $X(\mathbf{A})$ for each $x \in X(\mathbf{A})$. The direction $(\Leftarrow)$ is immediate.

Lemma 5.5. Let $\mathbf{A}$ be a modal Heyting algebra. Let $Y$ be an increasing closed subset of $X(\mathbf{A})$. For each $x \in X(\mathbf{A})$, if $R_{\square}^{\mathbf{A}}(x) \subseteq Y$, then $\max R_{\diamond}^{\mathbf{A}}(x) \subseteq Y$. Thus every $\square$-congruence is also a $\square \diamond$-congruence.

Proof. Assume that $R_{\square}^{\mathrm{A}}(x) \subseteq Y$. Let $y \in \max R_{\diamond}^{\mathrm{A}}(x)$. So by Lemma 3.2,

$$
y \in\left(R_{\square}^{\mathrm{A}} \cap R_{\diamond}^{\mathrm{A}} \subseteq^{-1}\right)(x) .
$$

Then there exists $z \in X(\mathbf{A})$ such that $(x, z) \in R_{\square}^{\mathbf{A}} \cap R_{\diamond}^{\mathbf{A}}$ and $y \subseteq z$. Since $y \in \max R_{\diamond}^{\mathbf{A}}(x)$, $z=y$. So, $y \in Y$.

Thus every $R_{\square}$-saturated subset is also an $R_{\diamond}$-saturated subset, and consequently $C_{R_{\square}}(X(\mathbf{A}))=C_{R}(X(\mathbf{A}))$. We conclude that $\operatorname{Con}(\mathbf{A})=\operatorname{Con}(\mathbf{A}, \square)$.

Remark 5.6. Let A be a modal Heyting algebra. Recall that a lattice filter $F$ is called an open filter if $\square a \in F$, when $a \in F$. It is easy to prove (see [19]) that the correspondence $F \rightarrow$ $\theta(F)$ is an isomorphism from the lattice of open filters of $\mathbf{A}$ onto the lattice of congruences of A. By Lemma 5.4 it follows that the lattice of open filters of $\mathbf{A}$ is isomorphic to the dual of the lattice of closed increasing subsets $Y$ of $X(\mathbf{A})$ such that $R_{\square}^{\mathbf{A}}(x) \subseteq Y$ for every $x \in Y$. By Lemma 5.5 it follows that the lattice $\operatorname{Con}(\mathbf{A}, \square)$ is isomorphic to the lattice $\operatorname{Con}(\mathbf{A})$. Thus, we have that the congruences with respect to $\square$ and $\diamond$ in a modal Heyting algebra are characterized by their open filters. But there exist $\diamond$-congruences in a modal Heyting algebra $\mathbf{A}$ that are not $\square$-congruences, and consequently are not characterized by an open filter. In other words, there exist increasing subsets $Y \subseteq X(\mathbf{A})$ that are $R_{\diamond}$-saturated of the Priestley space of $\mathbf{A}$ and are not $R_{\square}$-saturated.

Definition 5.7. Let $\mathbf{A}$ be a modal Heyting algebra. A lattice filter $F$ of $\mathbf{A}$ is a closed filter if $a \rightarrow b \in F$ implies $\diamond a \rightarrow \diamond b \in F$, for every $a, b \in A$. 
In the next result we will characterize the filters that correspond to $\diamond$-congruences in a modal Heyting algebra. As a consequence of this result, and taking into account that in a modal Heyting algebra $\mathbf{A}$ an open filter is also a closed filter, we get that in a modal Heyting algebra the lattice of $\diamond$-congruences is bigger than the lattice of its $\square$ congruences.

Proposition 5.8. Let $\mathbf{A}$ be a modal Heyting algebra. The lattice of $\diamond$-congruences of $\mathbf{A}$ is isomorphic to the lattice of closed filters of $\mathbf{A}$.

Proof. Let $F$ be a closed filter of $\mathbf{A}$. We prove that the increasing closed subset $Y_{F}$ is an $R_{\diamond}$-saturated subset of $X(\mathbf{A})$. Let $x \in Y_{F}$. Suppose that there exists $y \in \max R_{\diamond}(x)$ such that $F \varsubsetneqq y$. Then there exists $f \in F$ such that $f \notin y$. Let us consider the filter $[y \cup\{f\})$. Since $y \in \max R_{\diamond}(x)$,

$$
[y \cup\{f\}) \varsubsetneqq \diamond^{-1}(x) .
$$

Thus, there exist $q \in y$ and $d \in A$ such that $q \wedge f \leq d \in y$ and $\diamond d \notin x$. So, $f \leq q \rightarrow d \in F$. Since $F$ is a closed filter, $\nabla q \rightarrow \diamond d \in F \subseteq x$, and taking into account that $q \in y \subseteq \diamond^{-1}(x)$, we get that $\diamond d \in x$, which is a contradiction. Thus, $F \subseteq y$, and consequently $Y_{F}$ is an $R_{\diamond}$-saturated subset of $X(\mathbf{A})$.

Conversely, suppose that $Y_{F}$ is an $R_{\diamond}$-saturated subset of $X(\mathbf{A})$. Let $a \rightarrow b \in F$. If $\diamond a \rightarrow$ $\diamond b \notin F$, then there exists $x \in X(\mathbf{A})$ such that

$$
F \subseteq x, \quad \nabla a \in x, \quad \nabla b \notin x .
$$

So, there exists $y \in R_{\diamond}(x)$ such that $a \in y$. Hence there exists $m \in \max R_{\diamond}(x)$ such that $y \subseteq m$. Since $Y_{F}$ is an $R_{\diamond}$-saturated, and $F \subseteq x, F \subseteq m$. So, $a \rightarrow b \in m$. As $a \in y \subseteq m$, $b \in m$, which is a contradiction. Thus, $F$ is a closed filter.

Now, we will give the characterization of simple algebras. First we will give some auxiliary results.

Proposition 5.9. Let $\mathbf{A}$ be a $\square$-lattice. Let $R_{\square}=R_{\square}^{\mathbf{A}}$. Consider the following assertions:

(1) for all $x \in X(\mathbf{A}), \mathrm{Cl}\left(R_{\square}^{*}(x)\right)=X(\mathbf{A})$;

(2) for every $a \in A-\{1\}$ there exists $n \geq 0$ such that $\alpha_{n}(a)=0$.

Then (1) implies (2). If $\mathbf{A}$ is a $\square$-Heyting algebra, then (2) implies (1).

Proof. We prove that $1 \Rightarrow 2$. Let $a \in A-\{1\}$. Let us consider the set $H_{a}=\left\{\square^{n}(a): n \geq 0\right\}$ and let $F_{a}$ be the filter generated by $H_{a}$. Suppose that $0 \notin F_{a}$. Then there exists $x \in X(\mathbf{A})$ such that $F_{a} \subseteq x$. Since $\mathrm{Cl}\left(R_{\square}^{*}(x)\right)=X(\mathbf{A}), R_{\square}^{*}(x) \cap \sigma_{\mathbf{A}}(a)^{c} \neq \varnothing$. So there exists $y \in X(\mathbf{A})$ such that $y \in R_{\square}^{*}(x)$ and $a \notin y$. But this implies that $\alpha_{n}(a) \notin x$ for all $n \geq 0$, which is a contradiction. Thus there exists $n \geq 0$ such that $\alpha_{n}(a)=0$.

Assume that $\mathbf{A}$ is a $\square$-Heyting algebra. We prove $2 \Rightarrow 1$. Suppose that there exists $x \in$ $X(\mathbf{A})$ such that $\mathrm{Cl}\left(R_{\square}^{*}(x)\right) \neq X(\mathbf{A})$. Then there exists $y \in X(\mathbf{A})$ such that $y \notin \mathrm{Cl}\left(R_{\square}^{*}(x)\right)$. Since $\mathrm{Cl}\left(R_{\square}^{*}(x)\right)$ is a closed subset of $X(\mathbf{A})$, there exists $a, b \in A$ such that $R_{\square}^{*}(x) \cap \sigma_{\mathbf{A}}(a) \cap$ $\sigma_{\mathbf{A}}(b)^{c}=\varnothing$, that is,

$$
R_{\square}^{*}(x) \subseteq \sigma_{\mathbf{A}}(a) \Longrightarrow \sigma_{\mathbf{A}}(b)=\sigma_{\mathbf{A}}(a \longrightarrow b) .
$$


Thus $\square^{n}(a \rightarrow b) \in x$ for every $n \geq 0$. Then $\alpha_{n}(a \rightarrow b) \in x$ for every $n \geq 0$, which is a contradiction. Therefore $\mathrm{Cl}\left(R_{\square}^{*}(x)\right)=X(\mathbf{A})$.

Proposition 5.10. Let $\mathbf{A}$ be a $\square$-lattice. Let $R_{\square}=R_{\square}^{\mathrm{A}}$. Consider the following assertions:

(1) $\left\{x \in X(\mathbf{A}): \mathrm{Cl}\left(R_{\square}^{*}(x)\right) \neq X(\mathbf{A})\right\}$ is a closed increasing subset of $X(\mathbf{A})$ different from $X(\mathbf{A})$;

(2) there exists $a \in A-\{1\}$ such that for every $b \in A-\{1\}$ there exists $n \geq 0$ such that $\alpha_{n}(b) \leq a=\alpha_{n}(a)$.

Then (1) implies (2). If $\mathbf{A}$ is a $\square$-Heyting algebra, then (2) implies (1).

Proof. (1) $\Rightarrow(2)$. Let $Y=\left\{x \in X(\mathbf{A}), \mathrm{Cl}\left(R_{\square}^{*}(x)\right) \neq X(\mathbf{A})\right\}$. Since $Y \neq X(\mathbf{A})$, there exists $a \in$ $A-\{1\}$ such that $Y \subseteq \sigma_{\mathrm{A}}(a)$. Let $b \in A-\{1\}$. We prove that

$$
a \in F_{b}=\left[\left\{\square^{n} b: n \geq 0\right\}\right) .
$$

Suppose the contrary. Then there exists $y \in X(\mathbf{A})$ such that $F_{b} \subseteq y$ and $a \notin y$. Thus $y \notin Y$, and this implies that

$$
\mathrm{Cl}\left(R_{\square}^{*}(y)\right)=X(\mathbf{A}) .
$$

So $R_{\square}^{*}(y) \cap \sigma_{\mathrm{A}}(b)^{c} \neq \varnothing$ and consequently there exists $x \in R_{\square}^{*}(y)$ such that $b \notin x$. But, since $\alpha_{n}(b) \in y$ for every $n \geq 0$, then $b \in x$, which is a contradiction. Thus there exists $n \geq 0$ such that $\alpha_{n}(b) \leq a$.

We prove that $a \leq \alpha_{n}(a)$ for every $n \geq 0$. Let $x \in Y \subseteq \sigma_{\mathrm{A}}(a)$. We prove that

$$
R_{\square}^{*}(x) \subseteq \sigma_{\mathrm{A}}(a) .
$$

Let $y \in R_{\square}^{*}(x)$. Then $R_{\square}^{*}(y) \subseteq R_{\square}^{*}(x)$, and $\mathrm{Cl}\left(R_{\square}^{*}(y)\right) \subseteq \mathrm{Cl}\left(R_{\square}^{*}(x)\right) \neq X(\mathbf{A})$. Thus $y \in Y$ and hence $a \in y$.

Assume that $\mathbf{A}$ is a $\square$-Heyting algebra. We prove $2 \Rightarrow 1$. Let us consider the subset

$$
Y=\left\{x \in X(\mathbf{A}), \mathrm{Cl}\left(R_{\square}^{*}(x)\right) \neq X(\mathbf{A})\right\} .
$$

We prove that $Y=\sigma_{\mathbf{A}}(a)$. Let $x \in Y$. Since $\operatorname{Cl}\left(R_{\square}^{*}(x)\right) \neq X(\mathbf{A})$, there exists $z \in X(\mathbf{A})-$ $\mathrm{Cl}\left(R_{\square}^{*}(x)\right)$. So there exists $c, d \in A$ such that

$$
R_{\square}^{*}(x) \cap \sigma_{\mathbf{A}}(c) \cap \sigma_{\mathbf{A}}(d)^{c}=\varnothing .
$$

Since $\mathbf{A}$ is a Heyting algebra, $R_{\square}^{*}(x) \subseteq \sigma_{\mathbf{A}}(c \rightarrow d)=\sigma_{\mathbf{A}}(b)$. Thus $R_{\square}^{*}(x) \subseteq \sigma_{\mathbf{A}}\left(\alpha_{n}(b)\right)$ for all $n \geq 0$. If $x \notin \sigma_{\mathrm{A}}(a)=\sigma_{\mathrm{A}}\left(\alpha_{n}(a)\right)$, there exists $y \in R_{\square}^{*}(x)$ such that $a \notin y$. Since $y \in$ $R_{\square}^{*}(x) \subseteq \sigma_{\mathrm{A}}\left(\alpha_{n}(b)\right)$ for all $n \geq 0, \alpha_{n}(b) \in y$, and since $\alpha_{n}(b) \leq a$, we get $a \in y$, which is a contradiction. Thus $Y \subseteq \sigma_{\mathrm{A}}(a)$. 
18 Simple and subdirectly irreducibles bounded

Let $x \in X(\mathbf{A})$ such that $a \in x$. Then $\alpha_{n}(a) \in x$ for all $n \geq 0$. It follows that $R_{\square}^{*}(x) \subseteq$ $\sigma_{\mathrm{A}}(a)$. Since $a \neq 1$,

$$
\mathrm{Cl}\left(R_{\square}^{*}(x)\right) \subseteq \mathrm{Cl}\left(\sigma_{\mathrm{A}}(a)\right)=\sigma_{\mathbf{A}}(a) \neq X(\mathbf{A}) .
$$

Therefore $x \in Y$.

Theorem 5.11. Let $\mathbf{A}$ be a $\square$-Heyting algebra or a modal Heyting algebra. Let $R_{\square}=R_{\square}^{\mathbf{A}}$. Then the following conditions are equivalent:

(1) A is simple;

(2) for all $a \in A-\{1\}$ there exists $n \geq 0$ such that $\alpha_{n}(a)=0$;

(3) for all $x \in X(\mathbf{A}), \mathrm{Cl}\left(R_{\square}^{*}(x)\right)=X(\mathbf{A})$.

Proof. (1) $\Rightarrow(2)$. Let $a \in A-\{1\}$. Let us consider the filter

$$
F_{a}=\left\{y \in A: \alpha_{n}(a) \leq y \text { for some } n \geq 0\right\}
$$

It is easy to show that $F_{a}$ is a $\square$-filter and $F_{a} \neq\{1\}$. Since $\mathbf{A}$ is simple, $0 \in F_{a}$. So there exists $n \geq 0$ such that $\alpha_{n}(a)=0$.

$(2) \Rightarrow(1)$. Suppose that $\mathbf{A}$ is nonsimple. Then there exists a $\square$-filter $F$ such that $F \neq\{1\}$ and $F \neq A$. So there exists $x \in A-\{0,1\}$ such that $x \in F$. Since

$$
F_{x}=\left\{y \in A: \alpha_{n}(x) \leq y \text { for some } n \geq 0\right\} \subseteq F,
$$

$\alpha_{n}(x) \neq 0$ for all $n \geq 0$.

The equivalence between (2) and (3) follows by Proposition 5.9.

Corollary 5.12. Let $\mathbf{A}$ be a $\square$-Heyting algebra or a modal Heyting algebra. Let $R_{\square}=R_{\square}^{\mathrm{A}}$. Suppose that $A$ satisfies the inequality $\alpha_{n}(a) \leq \square^{n+1} a$. Then the following conditions are equivalent:

(1) A is simple;

(2) for all $a \in A-\{1\}, \alpha_{n}(a)=0$;

(3) for all $x \in X(\mathbf{A}),[x) \cup R_{\square}(x) \cdots \cup R_{\square}^{n}(x)=X(\mathbf{A})$.

Proof. It follows from Theorems 5.11 and 4.1 taking into account that $\alpha_{n}(a)=\alpha_{n+k}(a)$ for all $k \geq 0$.

In the next characterization of subdirectly irreducible algebras assertion (3) is new.

Theorem 5.13. Let A be a $\square$-Heyting algebra or a modal Heyting algebra. Let $R_{\square}=R_{\square}^{\mathrm{A}}$. Then the following conditions are equivalent:

(1) A is subdirectly irreducible;

(2) there exists $a \in A-\{1\}$ such that for all $b \in A-\{1\}$ there exists $n \geq 0$ such that $\alpha_{n}(b) \leq a=\alpha_{n}(a)$

(3) $\left\{x \in X(\mathbf{A}): \mathrm{Cl}\left(R_{\square}^{*}(x)\right) \neq X(\mathbf{A})\right\}$ is a closed and increasing subset of $X(\mathbf{A})$ different from $X(\mathbf{A})$.

Proof. The equivalence between (1) and (2) is proved in [19]. The equivalence between (2) and (3) follows by Proposition 5.10. 
Corollary 5.14. Let $\mathbf{A}$ be a $\square$-Heyting algebra or a modal Heyting algebra. Let $R_{\square}=R_{\square}^{\mathrm{A}}$. Suppose that A satisfies the inequality $\alpha_{n}(a) \leq \square^{n+1} a$. Then the following conditions are equivalent:

(1) A is subdirectly irreducible;

(2) there exists $a \in A-\{1\}$ such that for all $b \in A-\{1\}, \alpha_{n}(b) \leq a$;

(3) $\left\{x \in X(\mathbf{A}):[x) \cup R_{\square}(x) \cdots \cup R_{\square}^{n}(x) \neq X(\mathbf{A})\right\}$ is a closed and increasing subset of $X(\mathbf{A})$ different of $X(\mathbf{A})$.

Proof. It follows from Theorems 5.13 and 4.1 and taking into account that $\alpha_{n}(a)=\alpha_{n+k}(a)$ for all $k \geq 0$.

Finally, as a consequence of the previous results we have that a finite $\square$-Heyting algebra or a modal Heyting algebra $\mathbf{A}$ is simple if and only if there exists $n \geq 0$ such that $R_{\square}^{n}(x)=$ $X(\mathbf{A})$ for every $x \in X(\mathbf{A})$, and it is subdirectly irreducible if and only if there exists $x \in$ $X(\mathbf{A})$ such that $R_{\square}^{n}(x)=X(\mathbf{A})$ for some $n \geq 0$.

\section{Acknowledgment}

The author thanks the two referees for their comments and suggestions which helped to improve the previous version of this paper.

\section{References}

[1] R. Balbes and Ph. Dwinger, Distributive Lattices, University of Missouri Press, Missouri, 1974.

[2] G. Bezhanishvili, Varieties of monadic Heyting algebras. I, Studia Logica 61 (1998), no. 3, 367402.

[3] B. Birchall, Duality for distributive modal algebras, Master's thesis, ILLC, University of Amsterdam, Amsterdam, 2004.

[4] W. J. Blok and Ph. Dwinger, Equational classes of closure algebras. I, Indagationes Mathematicae 37 (1975), 189-198.

[5] M. Božić and K. Došen, Models for normal intuitionistic modal logics, Studia Logica 43 (1984), no. 3, 217-245.

[6] S. A. Celani and R. Jansana, A new semantics for positive modal logic, Notre Dame Journal of Formal Logic 38 (1997), no. 1, 1-18.

[7] _ Priestley duality, a Sahlqvist theorem and a Goldblatt-Thomason theorem for positive modal logic, Logic Journal of the IGPL 7 (1999), no. 6, 683-715.

[8] R. Cignoli, Distributive lattice congruences and Priestley spaces, Proceedings of the First "Dr. A. A. R. Monteiro" Congress on Mathematics (Spanish) (Bahía Blanca, 1991), Universidad Nacional del Sur, Bahía Blanca, 1991, pp. 81-84.

[9] _ Quantifiers on distributive lattices, Discrete Mathematics 96 (1991), no. 3, 183-197.

[10] J. M. Dunn, Positive modal logic, Studia Logica 55 (1995), no. 2, 301-317.

[11] M. Gehrke, H. Nagahashi, and Y. Venema, A Sahlqvist theorem for distributive modal logic, Annals of Pure and Applied Logic 131 (2005), no. 1-3, 65-102.

[12] R. Goldblatt, Varieties of complex algebras, Annals of Pure and Applied Logic 44 (1989), no. 3, $173-242$.

[13] P. R. Halmos, Algebraic logic. I. Monadic Boolean algebras, Compositio Mathematica 12 (1956), 217-249.

[14] Y. Hasimoto, Heyting algebras with operators, Mathematical Logic Quarterly 47 (2001), no. 2, 187-196. 


\section{Simple and subdirectly irreducibles bounded}

[15] J. C. C. McKinsey and A. Tarski, The algebra of topology, Annals of Mathematics. Second Series 45 (1944), 141-191.

[16] A. Petrovich, Distributive lattices with an operator, Studia Logica 56 (1996), no. 1-2, 205-224.

[17] V. Sofronie-Stokkermans, Duality and canonical extensions of bounded distributive lattices with operators, and applications to the semantics of non-classical logics. I, Studia Logica 64 (2000), no. 1, 93-132.

[18] _ Representation theorems and the semantics of non-classical logics, and applications to automated theorem proving, Beyond Two: Theory and Applications of Multiple-Valued Logic (M. Fitting and E. Orlowska, eds.), Studies in Fuzziness and Soft Computing, vol. 114, Physica, Heidelberg, 2003, pp. 59-100.

[19] F. Wolter, Superintuitionistic companions of classical modal logics, Studia Logica 58 (1997), no. 2, 229-259.

Sergio Arturo Celani: Consejo Nacional de Investigaciones Científicas Y Técnicas (CONICET) and Departamento de Matemáticas, Facultad de Ciencias Exactas, Universidad Nacional del Centro de la Provincia de Buenos Aires, Pinto 399, Tandil 7000, Argentina

E-mail address: scelani@exa.unicen.edu.ar 


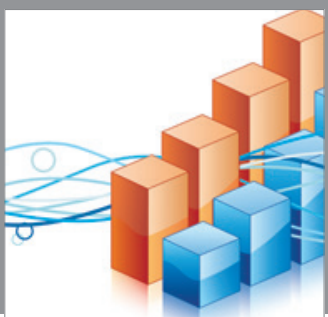

Advances in

Operations Research

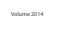

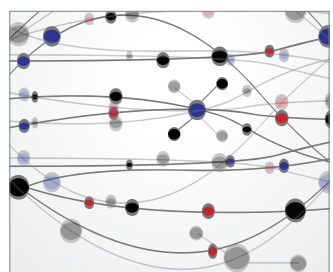

\section{The Scientific} World Journal
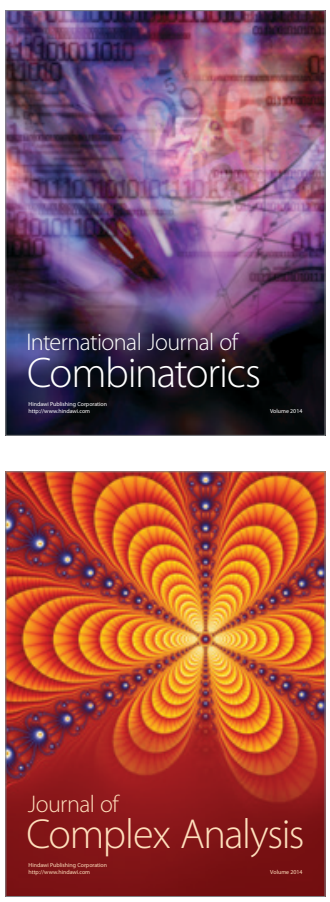

International Journal of

Mathematics and

Mathematical

Sciences
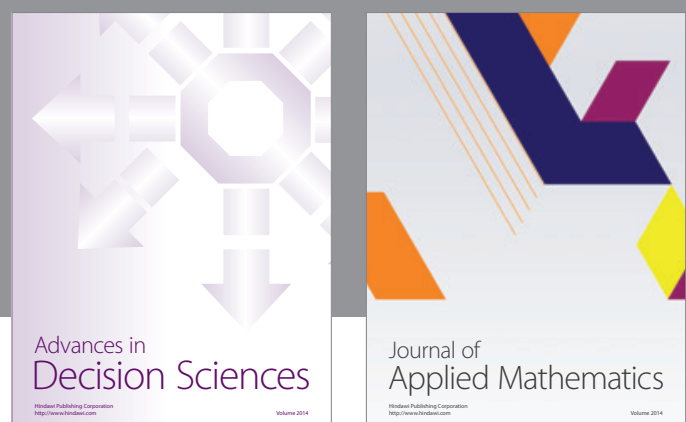

Journal of

Applied Mathematics
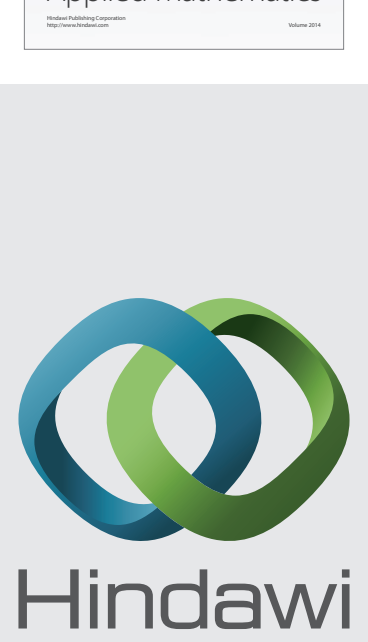

Submit your manuscripts at http://www.hindawi.com
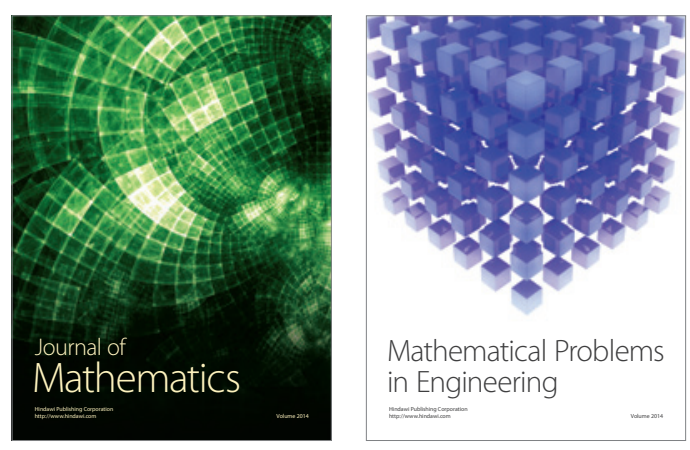

Mathematical Problems in Engineering
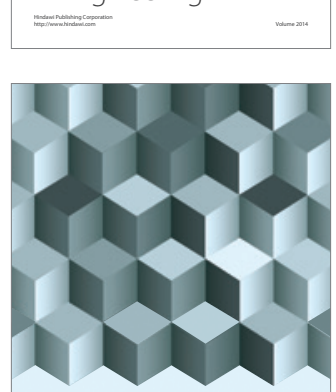

Journal of

Function Spaces
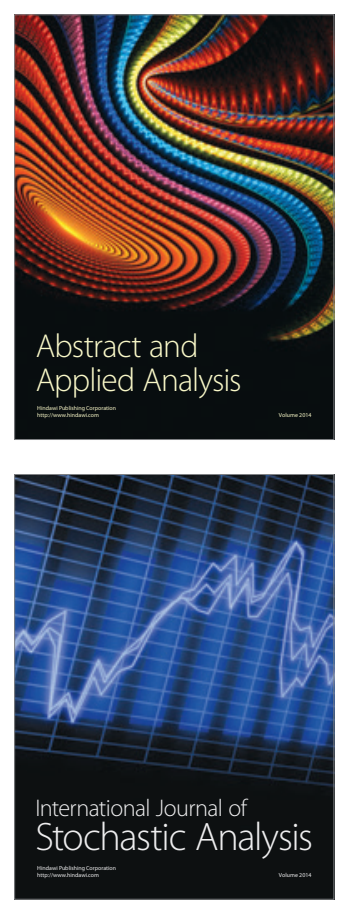

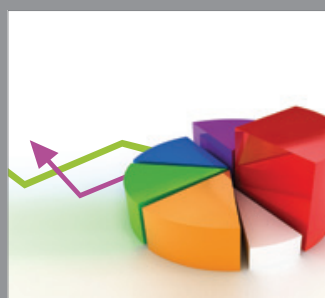

ournal of

Probability and Statistics

Promensencen
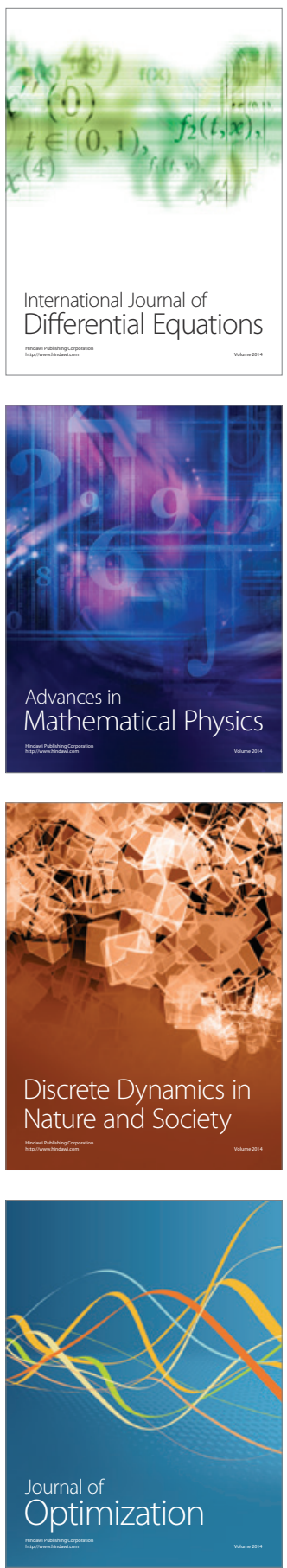